\title{
Design of UWB Monopole Antenna With EBG Structure And Ground With Rectangular Slots
}

\author{
Shiva Chauhan ${ }^{1}$ and P.K. Singhal ${ }^{2}$ \\ ${ }^{1,2}$ Department of Electronics Engineering, Madhav Institute of Technology and Science, Gwalior-474003, \\ M.P. , India \\ E-mail: ${ }^{1}$ shivachauhan6@gmail.com
}

\begin{abstract}
In this paper a low cost, simple and small size ultrawideiband circular monopole antenna is designed. The notch band is created by multiple number of slots in the ground plane. The proposed antenna is designed on the FR-4 substrate of height $1.6 \mathrm{~mm}$. The antenna is designed with ground plane of dimension $50 \times 55 \mathrm{~mm}$ and is fed by $50 \mathrm{ohm}$ microstrip line. It is found that the EBG design approach in the ground plane is a good candidate for frequency rejection of wide range. The antenna achieves an operational bandwidth of 1.5 Ghz (1.5 Ghz to $3 \mathrm{Ghz}$ ) which included ISM band (1.8 and 2.4 Ghz), WiFi / Bluetooth (2.4 Ghz), Satellite Radio (2.3 Ghz), and GPS (1.575 Ghz).
\end{abstract}

Keywords: Wireless Network, Hospital, Mobile Devices.

\section{INTRODUCTION}

Ultra wide band system have become more promising since they have low power consumption, low cost and capability of high data rate, low interference and low cost of installation when required for so many short range applications. Printed monopole antenna fabricated on substrate with modified ground plane provides large impedance bandwidth, which is useful for designing UWB microstrip antennas. In addition to UWB, Bluetooth applications have the advantage of license free applications in the ISM Band, which includes industrial, scientific and medical (2.4 $2.484 \mathrm{Ghz}$ band). Bluetooth is also one of the application, which used to transfer data between portable devices with high data rate [1].

Various methods have been proposed for Wideband characteristics. The conventional methods are cutting slots on the patch / ground plane or putting parasitic elements near to the radiator. Usually one notch or one slot is adequate for a notch band. The limitation of one notch is that they reject only one band. Due to the limitations of single-notch UWB antennas there is motivation to design dual / multinotches ultra wideband antennas. for multinotch / wide band multielements are commonly needed [3].

Recently Electromagnetic Band Gap (EBG) structure have been introduced and various types of EBG structures have been implemented in different applications such as reduction of surface waves, reduction of mutual coupling between two planer antennas. Since an EBG structure has a filtering behavior, it can be used to design UWB antennas with stop band characteristics [4].

The paper starts with the design of simple monopole antenna. The mushroom like EBG structure have been used in the same plane of monopole antenna for wide band response. The ground plane of antenna is designed with four rectangular slots of different length and width. The antenna radiates over the frequency band from 1.5 Ghz to $3 \mathrm{Ghz}$, which include different applications like ISM Band (industrial, scientific and medical applications), WiFi and Bluetooth, Cell phones, GPS, Microwave Oven and Satellite Radio.

The detail of antenna design with ultrawideband characteristics are given in the following sections. 
2

\section{ESIGN AND IMPLEMENTATION}

\subsection{EBG Structure}

The mushroom like EBG structure which we have studied before that it can provide a band gap characteristics. The EBG structure which we have used here is planer type of structure, which is based on usual mushroom type EBG structure.the difference between mushroom-like EBG structure and planer type of EBG structure is that in mushroom-like EBG structure there is a vias connecting patch to the ground plane, whereas in the planer type there is no connection i.e. no vias is connected.

It uses $1.6 \mathrm{~mm}$ thick FR-4 as a substrate. The metallic pattern i.e EBG structure is formed on the same plane, where antenna is designed while the other side metallic ground plane is designed. The structure does not used any vias to connect the patches with the ground plane, which makes the design simple and easy in fabrication. The unit cell dimension is $21.7 \mathrm{~mm} \times 17 \mathrm{~mm}$, which is shown below in Figure 1.

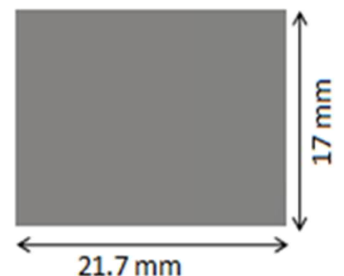

Fig. 1. unit cell of EBG structure

\subsection{UWB Monopole Antenna with Defected Ground Plane}

The configuration of proposed antenna is shown in Figure 2. The antenna is circular monopole antenna with defected ground plane. The antenna is the basic unit of our structure, which is attached to the EBG structure on the same plane. The antenna is fabricated on FR-4 substrate of thickness $1.6 \mathrm{~mm}$ and the relative permittivity of 4.4 . The width of a $50-\Omega$ microstrip feed line is $4 \mathrm{~mm}$. The size of the antenna is $50 \mathrm{mmx} 55 \mathrm{~mm}$.

The geometry of the circular monopole antenna with two cell of EBG structure along the feed line is shown in Figure 3.

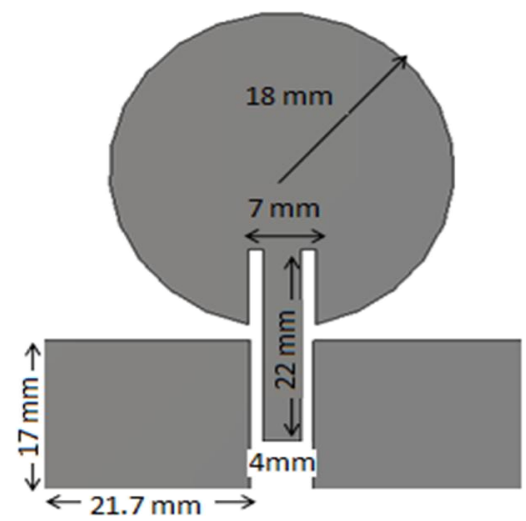

Fig. 2. Geometry of monopole antenna with $E B G$ structure

This antenna will be combined with a ground plane, which contain four rectangular slots. The structure of ground plane is shown in figure- 3 . The ground plane dimension is $50 \mathrm{~mm} \times 55 \mathrm{~mm}$.

The parameters of ground plane slots, the outer most first slot dimension is $50 \mathrm{~mm} \times 45 \mathrm{~mm}$, the second slot $40 \mathrm{~mm} \times 30 \mathrm{~mm}$, the third slot $23 \mathrm{~mm}$ $\mathrm{x} 20 \mathrm{~mm}$ and the inner most slot is $11 \mathrm{~mm} \times 10 \mathrm{~mm}$, which is shown below.

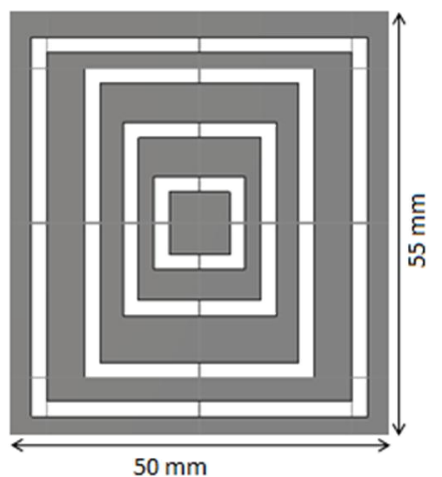

Fig. 3. geometry of ground plane with rectangular slots

The fabricated view of monopole antenna is shown in figure 4 and 5 . The fabrication is done, so that we can see the effect of environment and other parameters on the measurements. 
S. Chauhan and P.K. Singhal / International Journal of Computer Networks and Communications Security, 2 (6), June 2014

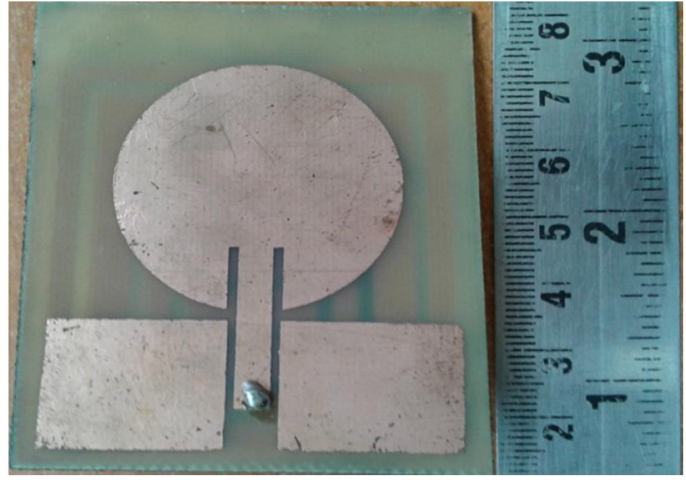

Fig. 4. Fabricated view of monopole antenna

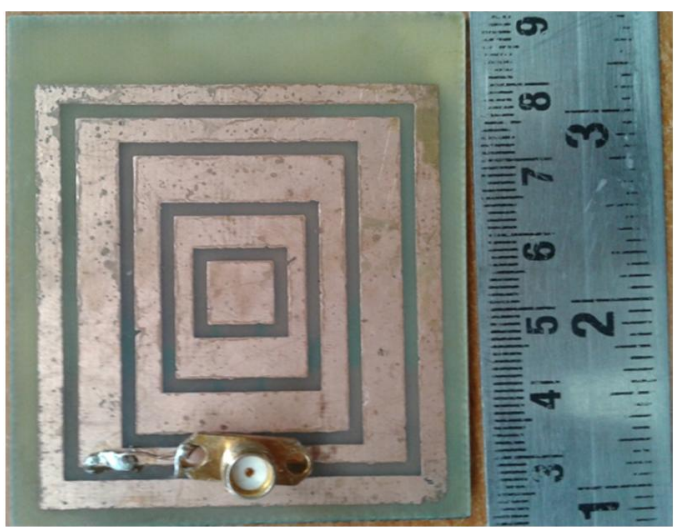

Fig. 5. Fabricated view of ground with rectangular slots

\section{RESULTS AND DISCUSSION}

The measured return loss characteristics of the fabricated monopole antenna has been shown in Figure 6.

The results shows the ultra wide band response. The $-10 \mathrm{~dB}$ return loss is for $1.5 \mathrm{Ghz}$ frequency band (1.5 Ghz to $3 \mathrm{Ghz})$. which means the $-10 \mathrm{~dB}$ bandwidth of the monopole antenna, which we have fabricated is $1.5 \mathrm{Ghz}$, this is because we named it UWB monopole antenna.

The wide stop band is created due to EBG structure and defected ground plane. The peak (negative) value of return loss is for $1.62 \mathrm{Ghz}$, which is $-48 \mathrm{~dB}$.

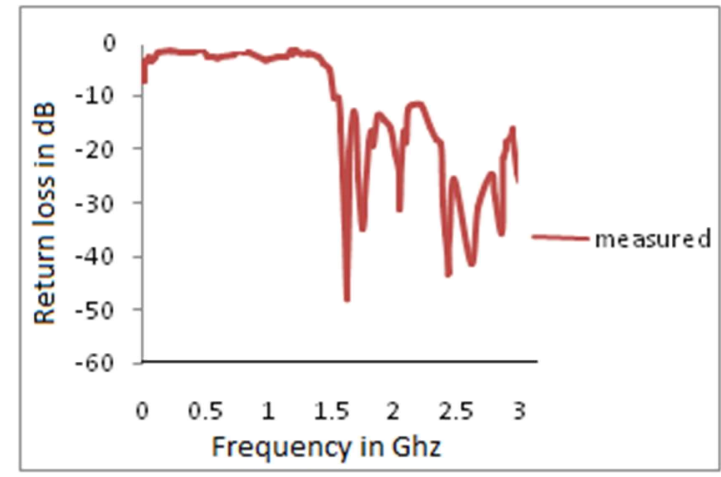

Fig. 6. Measured return loss of fabricated monopole antenna

The proposed monopole antenna with EBG structure and defected ground plane has been designed and optimized by CST microwave studio. CST microwave studio is used for calculating various parameters of antenna such as gain. Directivity, efficiency etc.

First we will discuss about the gain of the antenna. The gain of the monopole antenna is simulated using the far field pattern. The gain is calculated for the band from 1.5 Ghz to $3 \mathrm{Ghz}$, in which the gain is calculated for different frequencies. The maximum gain is aceived at frquency $1.62 \mathrm{Ghz}$, which is $4.628 \mathrm{~dB}$ and minimum is at $2.1 \mathrm{Ghz}$,which is $-2.69 \mathrm{~dB}$. So the gain variation is $7.318 \mathrm{~dB}$.

The variation of gain over the $1.5 \mathrm{Ghz}$ band (1.5 to $3 \mathrm{Ghz}$ ) is shown in figure-7.

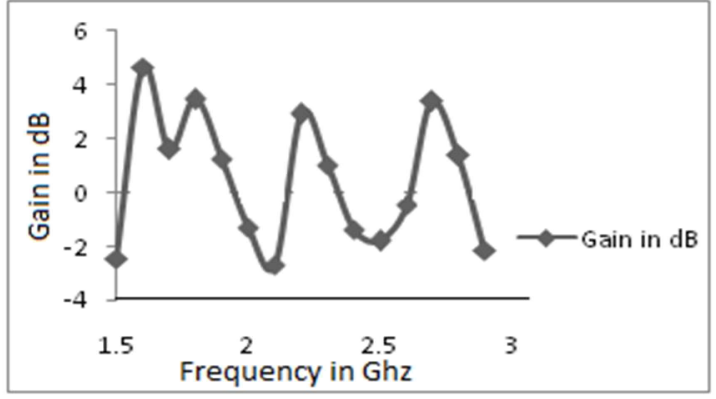

Fig. 7. variation of gain over the frequency range 
S. Chauhan and P.K. Singhal / International Journal of Computer Networks and Communications Security, 2 (6), June 2014

Now we will analyse how the directivity of the antenna varies over the freuency band of $1.5 \mathrm{Ghz}$. Figure- 8 shows the simulated directivity of monople antenna. The directivity of the antenna is $6.989 \mathrm{dBi}$ at the frequency $2.8 \mathrm{Ghz}$ and minimum at $2.1 \mathrm{Ghz}$, which is $3.77 \mathrm{Ghz}$.

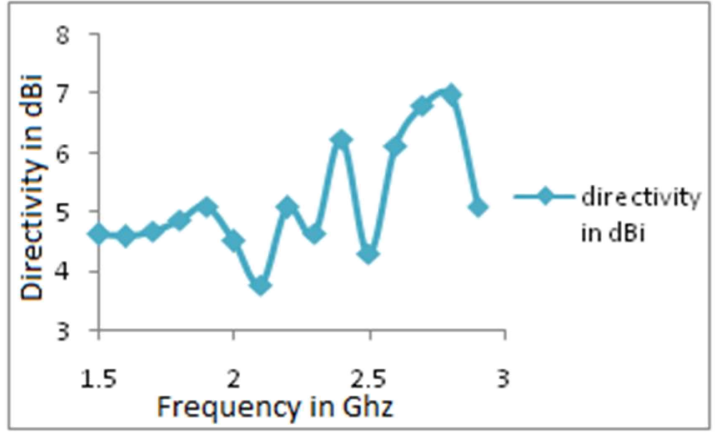

Fig. 8. variation of Directivity over the frequency band

Now we will characterized the efficiency of the moopole antenna. The effiency of the antenna determines which band is much suitable for practical applications. The maximum frequency comes about $90 \%$ for $1.62 \mathrm{Ghz}$. The efficiency graph of monopole antenna is shown in figure-9.

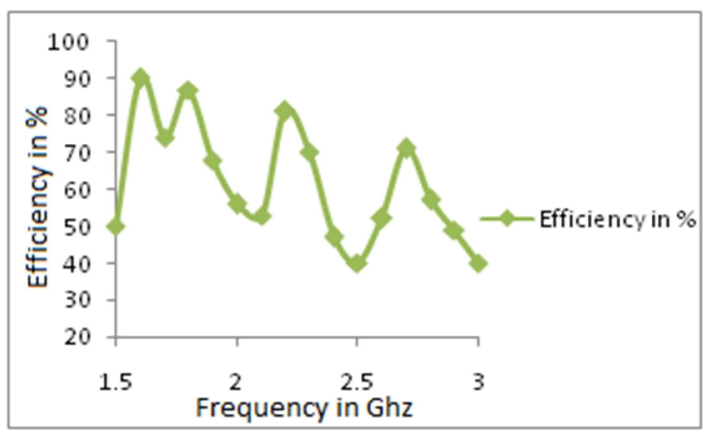

Fig. 9. Efficiency of monopole antenna

From all of the above results we can determine that between the $1.5 \mathrm{Ghz}$ to $3 \mathrm{Ghz}$ the best results for various parameters is $1.62 \mathrm{Ghz}$ and the gain and directivity shows its lowest value at $2.1 \mathrm{Ghz}$. So now we will determine the radiation pattern and polar plot for $1.62 \mathrm{Ghz}$ and $2.1 \mathrm{Ghz}$, so that we can compare the far field results, which is shown below.

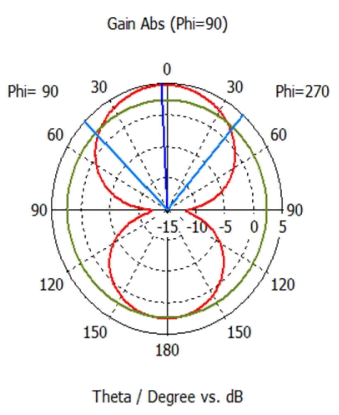

farfield ( $f=1.62)[1]$

Fig. 10. polar plot of monopole antenna at $1.62 \mathrm{Ghz}$

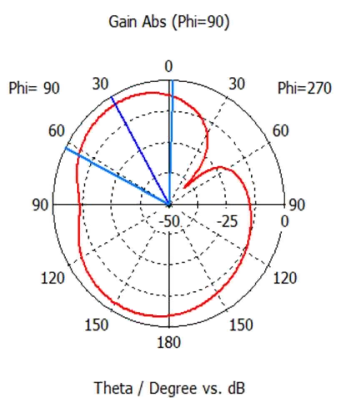

farfield $(f=2.1)[1]$

Fig. 11. polar plot of monopole antenna at $2.1 \mathrm{Ghz}$

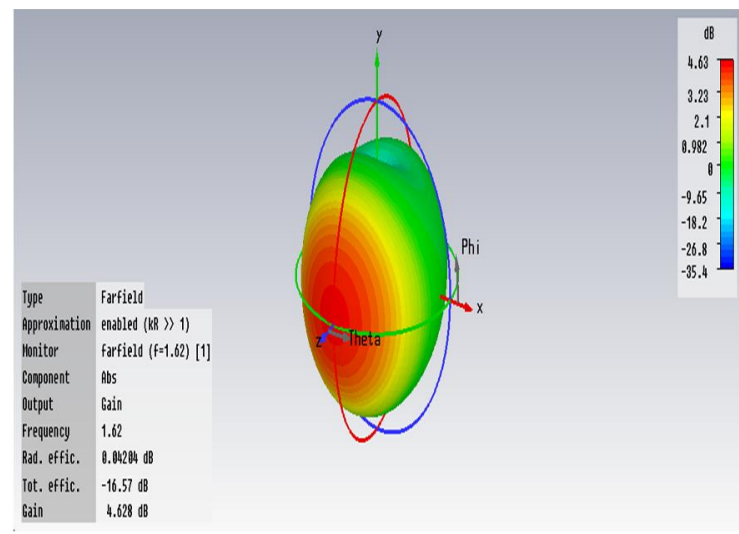

Fig. 12. 3D Far field Radiation pattern of antenna at $1.62 \mathrm{Ghz}$

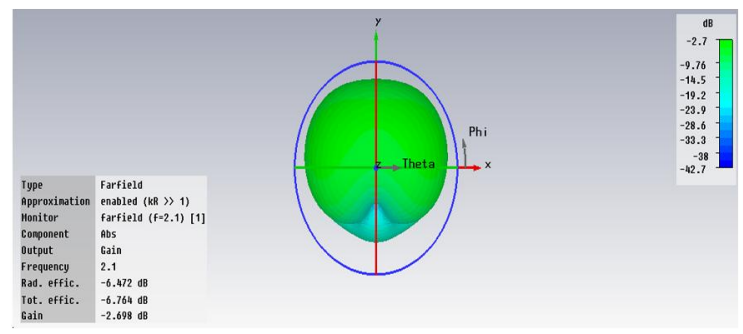

Fig. 13. 3D Far field radiation pattern of antenna at $2.1 \mathrm{Ghz}$ 


\section{CONCLUSION}

In this paper an approach to design the UWB antenna has been proposed. The design is simply placing the planer EBG structure near to the feed line of monopole antenna for create band gap also the ground plane is consisting of multiple number of slots for creating multiple number of notch band , which increase the overall bandwidth of antenna. Implementing the EBG structure in the vicinity of feed line does not change the behavior of radiating element and this is the advantage of proposed method. From the simulation results it can be concluded that the introduction of EBG structure has negligible effect on the various parameters of patch antenna. Therefore the proposed antenna is a good candidate for UWB applications including WLAN, Bluetooth, GPS and ISM band.

\section{REFERENCES}

[1] Gaurav K . Pandey, Hari S. Singh, Pradutt K . Bharti and Manoj K . Meshram " Design of stepped monopole UWB antenna with WLAN band notched using modified mushroom type EBG structure ", IEEE CONECCT 2013 1569684359

[2] S. Palreddy, A.I. Zaghloul and Y. Lee "An octave bandwidth Electromagnetic Bandgap (EBG) structure ", 6th European conference on Antennas and propogation (EUCAP).

[3] Lin Peng and Cheng-Li Ruan "UWB bandnotched monopole antenna design using Electromagnetic band gap structure ", IEEE transactions on microwave theory and techniques, VOL. 59, April 2011.

[4] Mohammad Yazdi and Nader Komjani "Design band-notched UWB monopole antenna by means of an EBG structure ", IEEE antennas and wireless propogation letters, VOL. 10, 2011.

[5] Nisar Ahmad Abbasi and Richard Langley " A small Quad-Band automotive antenna"

[6] Lin peng and Chengli Ruan " Design and time doamain analysis of compact multi band notched UWB antennas with EBG structures ", Progress in Electromagnetic Reaearch B, VOL. 47,339-357, 2013

[7] Son Trinh-Van and Chein Dao-Ngoc “ Dual band notch UWB antenna based on Electromagnetic band gap structures ", REV journal on Electronics and Communication, VOL.1, NO.2 April- June, 2011.

[8] Liyang, Mingyan fan, Fanglu Chen, Jingzhao She and Zhenghe Feng " A novel compact Electromagnetic band gap structure and its applications for mictowave circuits ", IEEE transactions on Microwave theory and techniques, VOL. 53, NO. 1, January 2005. 